Notas Clínicas

\title{
Dermatomiositis en el diagnóstico del cáncer: carcinoma de mama y de vesícula biliar
}

E. Martínez Ortega, A. Sánchez Muñoz, A. Ma García Tapiador, R. Dueñas García, A. Ma Lozano Barriuso

\section{Resumen}

La dermatomiositis (DM) es un raro síndrome paraneoplásico que se asocia al diagnóstico de diferentes tumores. Puede preceder a la enfermedad oncológica, cursar simultáneamente o incluso aparecer meses o años después de la misma. Presentamos dos casos de pacientes con DM asociada a carcinoma de mama y de vesícula biliar, describiéndose los principales puntos de interés que esta entidad clínico-patológica pueda tener para el oncólogo.

\section{Palabras clave:}

Dermatomiositis. Síndrome paraneoplásico. Cáncer de mama. Cáncer de vesícula biliar.

Oncología, 2005; 28 (4):193-196

Servicio de Oncología Médica 


\section{Summary}

Dermatomyositis (DM) is a rare paraneoplastic syndrome associated with different tumors. It can precede the tumor appearance or go on simultaneously or even appear years after the tumor is diagnosed. We report two cases of patients with DM associated with breast cancer and gallbladder cancer, and make a review of the main interesting points for oncologists.

Key words: Dermatomyositis. Paraneoplastic syndrome. Breast cancer. Gallbladder cancer.

\section{Introducción}

La dermatomiositis (DM) es un raro síndrome paraneoplásico que se asocia al diagnóstico de diferentes tumores. Puede preceder a la enfermedad oncológica, cursar simultáneamente o incluso aparecer meses o años después de la misma. Presentamos dos casos de pacientes con DM asociada a carcinoma de mama y de vesícula biliar, describiéndose los principales puntos de interés que esta entidad clínico- patológica pueda tener para el oncólogo.

\section{Casos clínicos}

Paciente mujer de 57 años sin antecedentes de interés, que consultó en febrero de 2000 por cuadro de tres meses de evolución de enrojecimiento facial de predominio matutino, edemas en miembros superiores, disfagia, disfonía, astenia y febrícula. A la exploración física se objetivó edema facial y eritema de predominio en región malar. Las pruebas analíticas realizadas estaban dentro de los rangos de la normalidad.

En mayo de 2000, comenzó con mialgias generalizadas y una discreta elevación de la enzima creatinquinasa (CK) de $247 \mathrm{U} / \mathrm{L}(0-167 \mathrm{U} / \mathrm{L})$. Ante la sospecha clínica de DM se realizaron un electromiograma y una biopsia de la piel afectada, que confirmaron el diagnóstico. El TAC de tórax y abdomen, enema opaco y marcadores tumorales no presentaban alteraciones significativas. Tras un empeoramiento clínico se inició tratamiento con prednisona a dosis de $1 \mathrm{mg} / \mathrm{Kg}$, con mejoría sintomática. En noviembre de 2000, apareció una tumoración dolorosa en el cuadrante súpero-externo de la mama derecha. En una mamografía se apreciaba un nódulo de características radiológicas sugestivas de malignidad. Se intervino mediante tumorectomía con posterior vaciamiento axilar. El estudio anatomopatológico informó de carcinoma ductal infiltrante pobremente diferenciado de $1.5 \mathrm{~cm}$ de tamaño. Se aislaron 21 adenopatías sin infiltración tumoral (pT1N0Mo), con receptores hormonales negativos.

Se inició tratamiento con quimioterapia adyuvante esquema CMF (ciclofosfamida $600 \mathrm{mg} / \mathrm{m}^{2}$, metotrexate $40 \mathrm{mg} / \mathrm{m}^{2}, 5-\mathrm{FU} 600 \mathrm{mg} / \mathrm{m}^{2}$, días 1 y 8 , cada 4 semanas) por 6 ciclos, seguido de RT y revisiones periódicas posteriores.

Desde febrero de 2004 ha reaparecido la clínica de DM sin encontrarse hasta el momento actual evidencia de enfermedad, continuándose un seguimiento estrecho de la paciente ante la posibilidad de recurrencia tumoral o de un segundo tumor.

Paciente varón de 53 años con antecedentes de hipertensión arterial, que consultó en agosto de 2000 por eritema facial y cervical. En octubre del mismo año comenzó con dolor en musculatura proximal de miembros superiores e inferiores y debilidad progresiva con limitación de la capacidad funcional. A la exploración física se evidenció una erupción facial de coloración violácea, edema periorbitario, telangiectasias malares y exantema periungueal (Fig. 1). Se solicitó electromiograma del músculo deltoides con patrón de características miopáticas, y una biopsia de la piel afectada compatible con dermatomiositis (Fig. 2). Se realizó un estudio del paciente mediante TAC toraco-abdominal, RMN abdómino-pélvica y endoscopia digestiva alta y baja que fueron normales. En la analítica general se evidenciaron ci- 


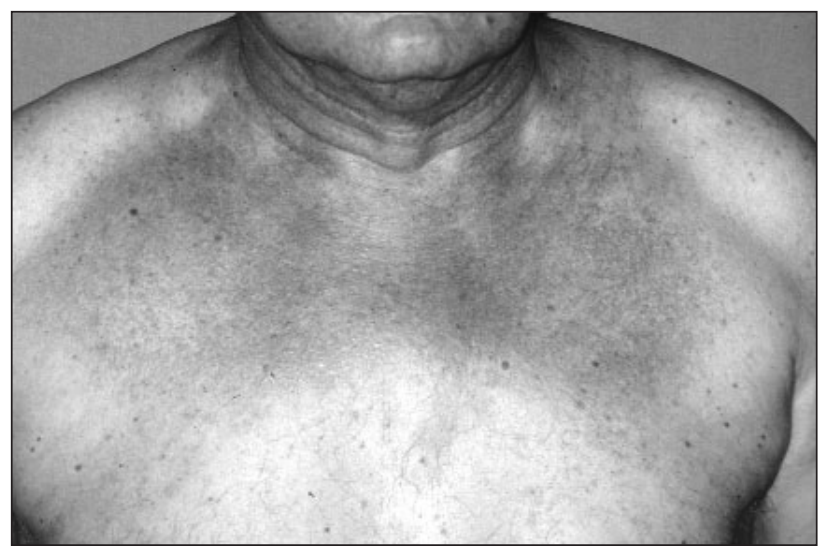

Figura 1.

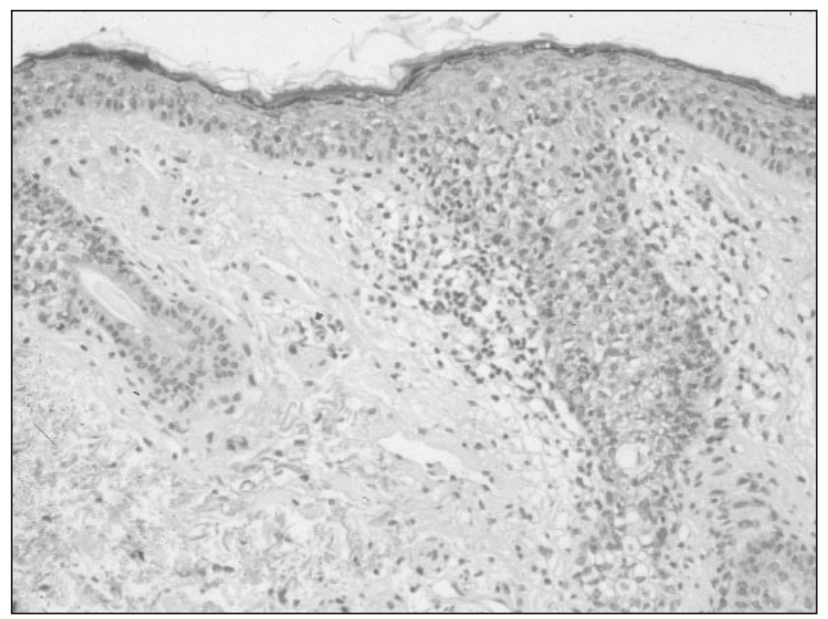

Figura 2.

fras de CPK de 23.256 U/L (0-240 U/L), LDH de $3.083 \mathrm{U} / \mathrm{L}(0-250 \mathrm{U} / \mathrm{L})$ y niveles de CA $19.9 \mathrm{de}$ $55^{\prime} 79 \mathrm{U} / \mathrm{ml}(0-37 \mathrm{U} / \mathrm{ml})$. Se pautó tratamiento con prednisona a dosis de $1 \mathrm{mg} / \mathrm{Kg}$ con disminución progresiva de dichas enzimas y mejoría de la sintomatología.

El enfermo siguió revisiones periódicas. En mayo de 2001 una RMN de abdomen y colonoscopia fueron normales, y en una analítica general solo destacaba un CA 19.9 de $385 \mathrm{U} / \mathrm{ml}$; la CK permaneció normal con corticoides de mantenimiento. En enero de 2003, una nueva RMN abdomino-pélvica describía una vesícula biliar filiforme sin significado patológico. Los niveles de CA 19.9 eran >5.000 U/ml. TAC de tórax con infiltrado intersticial en parénquima pulmonar sugerente de neumonitis asociada a dermatomiositis. En agosto del 2003, la RMN abdo- minal mostró una tumoración sugerente de proceso tumoral de vesícula biliar con infiltración del parénquima hepático adyacente, con cifras de CA 19.9 $>5.000 \mathrm{U} / \mathrm{ml}$.

El tumor se consideró irresecable durante el acto quirúrgico. Se tomaron muestras para estudio anatomopatológico, que se informaron como adenocarcinoma bien diferenciado de vesícula biliar y metástasis hepáticas

El paciente presentó un rápido deterioro de su estado general y de su función hepática y se pautaron medidas sintomáticas paliativas.

\section{Discusión}

La polimiositis es un síndrome paraneoplásico que causa un proceso inflamatorio a nivel muscular. Cuando se acompaña de manifestaciones cutáneas características constituye la dermatomiositis. Cursa con clínica de debilidad muscular generalizada y dolorosa de predominio proximal a nivel de extremidades, asociada a trastornos cutáneos característicos en regiones malares, periorbitarias y parte superior del cuello fundamentalmente. Su diagnóstico se realiza mediante una biopsia muscular que describe una necrosis en células musculares y la presencia de infiltrado perifascicular y perivascular, y un electromiograma con patrón miopático. El marcador más sensible es la enzima CK, ya que su elevación está presente en el $90 \%$ de los enfermos.

La relación entre DM y cáncer ha sido descrita en diferentes estudios retrospectivos ${ }^{1-5}$. Se estima que la incidencia de cáncer en pacientes con DM es entre un 15-30\% del total de los casos ${ }^{5}$.

Los tumores a los que se asocia con más frecuencia son el cáncer de mama, de ovario, gastrointestinales, pulmón y linfomas, variando su frecuencia en las series revisadas. Son muy pocos los casos descritos en la literatura de DM asociada a tumores de la vesícula biliar. Únicamente hemos encontrado dos casos en la revisión realizada ${ }^{4,6}$. La DM puede preceder a la enfermedad oncológica, cursar simultáneamente o incluso aparecer meses o años después de la misma. En los dos casos presentados, el diagnóstico de DM precedió en 6 y 36 meses al cáncer de mama y de vesícula biliar, respectivamente. En pacientes mayores de 50 años con cuadro de DM es aconsejable descartar una posible neoplasia oculta y 
realizar un seguimiento periódico ${ }^{7}$. Las recomendaciones en cuanto a las pruebas de screening son muy variables, desde la anamnesis y la exploración física exhaustiva exclusivamente, hasta la realización de maniobras invasivas y pruebas de imagen de forma rutinaria $^{5}$. El tiempo de mayor riesgo de aparición de una neoplasia tras el diagnóstico de DM, no está bien definido, estableciéndose entre uno y cinco años según las distintas series ${ }^{1,3,4}$. La asociación temporal entre cáncer y DM es de gran utilidad. Se han descrito casos de mejoría clínica de DM seguida de una respuesta al tratamiento del cáncer, así como casos de exacerbación de la sintomatología coincidiendo con una recurrencia o progresión tumoral ${ }^{8}$. Actualmente nuestra primera paciente sigue revisiones periódicas estrechas para detectar de modo precoz tanto una posible recidiva como un segundo tumor que pudieran acontecer, si bien el valor que ello pueda tener en cuanto a su pronóstico es incierto.

El tratamiento de la DM paraneoplásica incluye la administración de corticoides a dosis de 1 a $2 \mathrm{mg} / \mathrm{kg}$ al día, disminuyendo la dosis conforme mejoran los síntomas, además del tratamiento de la enfermedad tumoral de base.

La supervivencia a 5 años en enfermos con DM oscila entre $79 \%$ y $57 \%$ según las distintas series. Se estima un porcentaje de supervivencia a los 2 años del $25 \%$ en los casos de DM asociada a cáncer, y del $57 \%$ a los 3 años en el resto de los casos ${ }^{9}$.

Diversos autores han intentado determinar factores predictivos de malignidad de DM tales como la exacerbación de una neumonitis intersticial, lo cual sucedió en nuestro segundo caso, la determinación de niveles bajos en suero de CPK, necrosis cutánea asociada y aumento en la VSG > $40 \mathrm{~mm} / 1^{\mathrm{a}} \mathrm{h}$, aunque los resultados son poco concluyentes. Serían de gran utilidad futuras investigaciones dirigidas a determinar autoanticuerpos relacionados con esta patología, como en el caso de otros síndromes paraneoplásicos, de cara a su diagnóstico y a predecir mejor el pronóstico de la enfermedad ${ }^{7}$.

\section{Bibliografía}

1. Sigurgeirsson B, Lindelof B, Edhag O, Allander E. Risk of cancer in patients with dermatomyositis or polymyositis. A population-based study. N Engl J Med 1992; 326:363-367.

2. Chow WH, Gridley G, Mellemkjaer L, el al. Cancer risk following polymyositis and dermatomyosytis: a nationwide cohort study in Denmark. Cancer Causes Control 1995; 6:913.

3. Zantos D, Zhang Y, Felson D. The overall and temporal association of cancer with polymyositis and dermatomyositis. J Rheumatol 1994; 21:1855-1859.

4. Ario A, Pukkala E, Isomaki H. Elevated cancer incidence in patients with dermatomyositis: a population based study. J Rhematol 1995; 22:1300-1303.

5. Hill CL, Zhang Y, Sigurgeirsson B, et al. Frequency of specific cancer types in dermatomyositis and polymyositis; a population-based study. Lancet 2001; 357:96-100.

6. Yiannopoulos G, Ravazoula P, Meimaris N, et al. Dermatomyositis in a patient with adenocarcinoma of the gal bladder. Ann Rheum Dis 2002; 61:663-664.

7. Wakata N, Kurihara T, Saito E, Kinoshita M. Polymyositis and dermatomyositis associated with malignancy: a 30-year retrospective study. Intern J Dermatol 2002; 41:729-734.

8. Goyal S, Nousari HC. Paraneoplastic amyopatic dermatomyositis associated with breast cancer recurrence. J Am Acad Dermatol 1999; 41:874-875.

9. Basset-Seguin N, Roujeau JC, Gherardi R, et al. Prognostic factors and predictive signs of malignancy in adult dermatomyositis. A study of 32 cases. Arch Dermatol 1990;126: 633-637.
Correspondencia:

Dra. E. Martínez Ortega

Servicio de Oncología Médica

Complejo Hospitalario de Jaén

Avda. Ejército Español, 10

E-23001 Jaén

E-mail: Esthermartinezz@yahoo.es 